\title{
People's Interactions with Cognitive Assistants for Enhanced Performances
}

\author{
Md Abul Kalam Siddike \\ Japan Advanced Institute of \\ Science and Technology \\ kalam.siddike@gmail.com
}

\author{
Jim Spohrer \\ IBM Research \\ spohrer@us.ibm.com \\ Youji Kohda
}

\author{
Haluk Demirkan \\ University of Washington- \\ Tacoma \\ haluk@uw.edu
}

Japan Advanced Institute of Science and Technology

kohda@jaist.ac.jp

\begin{abstract}
When cognitive computing enabled smart computers are growing in our daily lives, there are not many studies that explain how people interact and utilize these solutions, and the impact of these smart machines to people's performance to do things. In this paper, a theoretical framework for boosting people's performance using cognitive assistants (CAs) was developed and explained using the data analysis from 15 interviews. The results show that people interaction with CAs enhance their levels of cognition and intelligence that help them to enhance their capabilities. Enhanced capabilities help people to enhance their performance.
\end{abstract}

\section{Introduction}

There is no doubt that computers are increasingly capable of doing things that make humans more knowledgeable and productive. Today smart machines are able to recognize patterns, perform rule-based analysis from very large amounts of data, solve both structured and unstructured problems, recognize voices, process natural language, learn, and interact with other computers and humans.

Apple's Siri, Google's Now, Amazon's Echo, IBM's Watson (Figure 1) and many other smart machines, cognitive tools, are beginning to reach a level of utility that provide a foundation for a new generation of assistants, cognitive assistants (CAs) [24]. CAs are new decision support tools that are able to augment human capabilities and expertise [21] [28] [30].

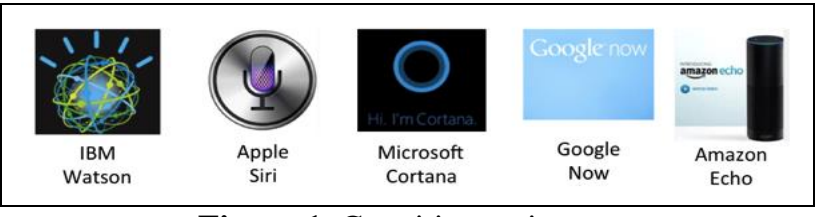

Figure 1. Cognitive assistants
CAs can provide people high-quality recommendations and help them make better data driven decisions [4]. People problem solving capabilities significantly augmented by the interaction of people and CAs [24] [26]. Cognitive computing and sensor technologies have begun to emerge to augment and scale the capabilities of people in specific ways [11] [16]. Smart machines can potentially progress from cognitive tools to assistants to collaborators to coaches, and be perceived differently depending on the role that they play in a service system [21].

People have limited life spans and limited cognitive capabilities, suffering from "bounded rationality" in decision making [20]. As knowledge accumulates in society, Jones [10] identified the condition known as the "knowledge burden". In addition, there is a "halflife of knowledge" in any innovation oriented society [1]. Furthermore, today people flooded with data, information and knowledge [2] [14] [31]. To address some of these challenges, researchers have been working on "knowledge factories" known for teaching (learning), discovery (research) and application of knowledge (entrepreneurship and policy making) [29]. People also focus on acquiring special type of knowledge from experience and a sense of humor to cope with life's challenges. When data is growing exponentially, people need more resilient and sustainable approaches to address those challenging problems. Today for example, cognitive computing have begun to emerge to augment and scale the capabilities of people in specific ways [11] [16]. With cognitive assistants, people has an opportunity to analyze large amount of data very fast, and make much more efficient, effective and accurate decisions.

The main motivation of this paper laid down by Douglas Engelbart, an American engineer, and an early computer and Internet pioneer who invented the computer mouse, urged people to work quickly "to augment human intellect and address complex, urgent problems" [6]. Technology and organizations are two 
instruments that people have developed to augment their intellect in order to enhance their performance [15]. Enhancing human performance means enhancing human abilities to achieve goals both individually and collectively through the convergence of technologies. Enhancing human performance also hold the promise to make people healthier, wealthier, and wiser as well as to make business more responsive, resilient, and adaptive [22].

Table 1. Sample list of cognitive assistants

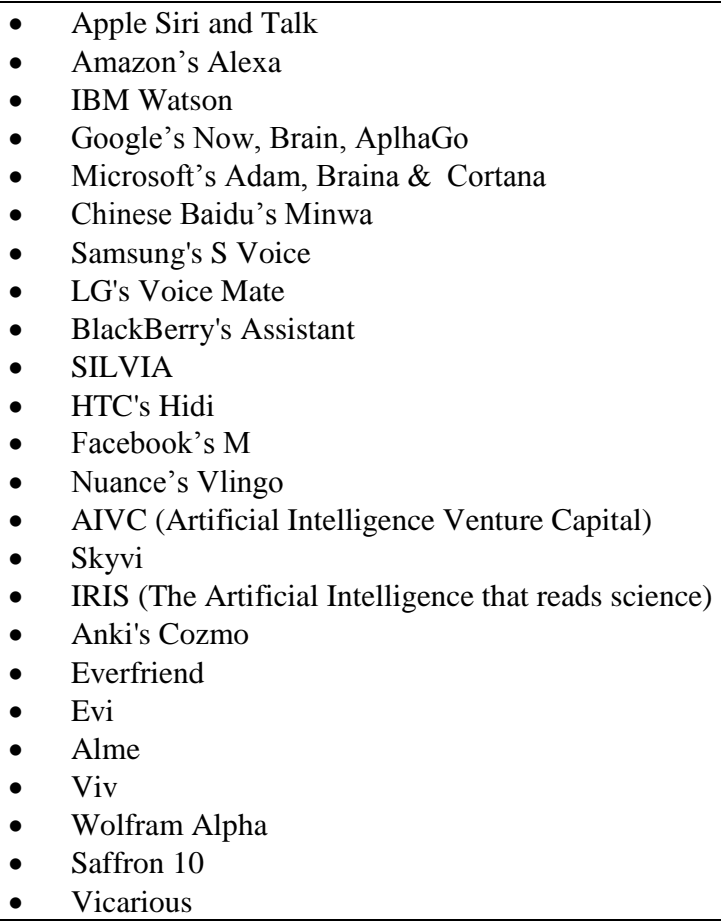

Cognitive assistants have potentials to improve the efficiency and effectiveness of people's decisions. CAs work via:

- Text (online chat), especially in an instant messaging app or other app

- Voice, for example with Amazon Alexa on the Amazon Echo device, or Siri on an iPhone

- By taking and/or uploading images, as in the case of Samsung Bixby on the Samsung Galaxy S8

Some of them are accessible via multiple methods, such as Google Assistant via chat on the Google Allo app and via voice on Google Home smart speakers. Virtual assistants use natural language processing to match user text or voice input to executable commands. Many continually learn using artificial intelligence techniques including machine learning ${ }^{1}$.

More and more companies are working on cognitive assistants - and each month a new company

${ }^{1}$ https://en.wikipedia.org/wiki/Virtual_assistant_(artificial_intelligen ce) shows up working on their version of an intelligent personal assistant. When the list of cognitive assistants in the market is growing exponentially every day (Table 1), there are not many studies that explain how people interact with these smart machines and how people's abilities and capabilities are enhanced. Table 2 shows features of some CAs.

The purpose of this paper is to understand people's interactions with smart machines, and to develop a framework to boost people's performance through the interaction with cognitive assistants.

The rest of the paper is organized as following. The next section provides more details on cognitive assistants and how they work. Section 3 describes the proposed framework that explains how people's performance can be increased with CAs. In the section 4, we explain our research methodology further. We conclude with implications of this research to practice and possible future projects.

\section{Cognitive Assistants (CAs)}

CAs are emerging decision support tools. They are able to augment human capabilities and expertise by understanding the environment around us with a depth and clarity [21] [28] [30]. Enabled by large data sets and speedy computers, CAs can provide people with high-quality recommendations and can help them make better data driven decision [4]. People problem solving capabilities can be significantly augmented by the interaction of people and CAs [24] [26]. CAs could be different formats such as speech (conversational agents), typing, and gestures (real-robots), and they can augment the cognitive and social capabilities of people. Today most cognitive computing and sensor technologies have begun to emerge to augment and scale the capabilities of people in specific ways [11] [16]. Cognitive systems can potentially progress from tools to assistants to collaborators to coaches and be perceived differently depend on the role that they play in a service system (Table 2 ).

To be people-centered, this progression requires that cognitive systems recursively acquire more advance models of their users in order to develop expert cognitive and social capabilities. Eventually, CAs will exist for all types of occupations and societal roles in service systems - and this will be the dawn of the era of smart, people-centered service systems. The ownership of cognitive systems and the personal data on which they will operate- as they build user models - will become an active area of legislation in coming years, as companies that produce intelligent personal assistants seek to monetize fully the benefits they create for customers. 
Table 2: Features of some CAs

\begin{tabular}{|c|c|c|}
\hline Name of CAs & Functionalities & Platforms used by CAs \\
\hline Apple Siri & $\begin{array}{l}\text { Siri can help get things done-like } \\
\text {-sending messages, placing calls, and making dinner } \\
\text { reservations } \\
\text {-show the best route home } \\
\text {-set alarm and ask questions } \\
\text {-user voice be the remote control for connected } \\
\text { products } \\
\text {-can be tuned in the world working with Wikipedia, } \\
\text { Yelp and other online service for getting more answers }\end{array}$ & $\begin{array}{l}\text {-Apple's platform } \\
\text {-Wikipedia } \\
\text {-Yelp }\end{array}$ \\
\hline IBM Watson & $\begin{array}{l}\text {-Watson Engagement Advisor interacts with } \\
\text { customers, listens to questions and offers solutions } \\
\text {-Watson Explorer accesses and analyzes structure and } \\
\text { unstructured content } \\
\text {-Watson Discovery Adviser makes connections and } \\
\text { draws relationships between data for everyone's } \\
\text { activities } \\
\text {-Watson Oncology analyzes a patient's medical } \\
\text { information against a vast array of data and expertise to } \\
\text { provide evidence-based treatment } \\
\text {-Watson for Clinical Trail Matching enhances } \\
\text { clinicians' ability to more easily find the potential list } \\
\text { of clinical traits. }\end{array}$ & IBM Bluemix \\
\hline $\begin{array}{l}\text { Google's Now, } \\
\text { Brain, AlphaGo, } \\
\text { Home, Assistant }\end{array}$ & $\begin{array}{l}\text {-answering questions, make recommendations, perform } \\
\text { actions by delegating requests to a set of web services } \\
\text {-Google Assistant is the evolution of Google Now and } \\
\text { it can engage in two-way dialogue with the users (voice } \\
\text { and chat) } \\
\text {-Google Home-a voice-activated home product, } \\
\text { allows family to get answers from Google, stream } \\
\text { music and mange everyday tasks }\end{array}$ & Google' platform \\
\hline $\begin{array}{l}\text { Amazon's } \\
\text { Alexa }\end{array}$ & $\begin{array}{l}\text { It is capable of voice interaction; music playback; } \\
\text { making to-do lists; setting alarms; streaming podcasts; } \\
\text { playing audiobooks; providing weather, traffic and } \\
\text { other real time information; and can also control } \\
\text { several smart devices using itself as a home automation } \\
\text { hub }\end{array}$ & Amazon's platform \\
\hline $\begin{array}{l}\text { Microsoft's } \\
\text { Adam, Braina \& } \\
\text { Cortana }\end{array}$ & $\begin{array}{l}\text { It can set reminders, speak out our mind, use maps, } \\
\text { dictate emails, search terms, show flight information, } \\
\text { and provide news feeds }\end{array}$ & Microsoft's platform \\
\hline Viv & $\begin{array}{l}\text {-can accommodate external plug-ins written to work } \\
\text { with the assistant } \\
\text {-can also handle more complex queries }\end{array}$ & Viv's platform \\
\hline
\end{tabular}

Table 3 summarizes the progression from cognitive tools, to assistants, collaborators and coach, and eventually trusted cognitive mediators. In the future, cognitive mediators might be able to assist people boost the creativity and productivity of their interactions, and deal with complexity and the pace of change in a world in which data is a growing resource for all. Cognitive mediators will also have a great deal of information about the person they are bound to, including memories of all experiences and interactions.

When people change employment from one company to another, governance policies that allow a cognitive mediator to forget confidential information will be required. 
Table 3. Progression of Tools-assistants-collaboratorcoach

\begin{tabular}{|l|l|}
\hline Nature & What will do for us \\
\hline Tools & $\begin{array}{l}\text { Data and information (as a tool will be } \\
\text { able to process trillions of data and } \\
\text { information) }\end{array}$ \\
\hline Assistant & $\begin{array}{l}\text { Knowledge (as an assistant cognitive } \\
\text { mediator will have more knowledge } \\
\text { about people) }\end{array}$ \\
\hline Collaborator & $\begin{array}{l}\text { Understanding as a collaborator can } \\
\text { understand people's situation and } \\
\text { culture, conditions more than us) }\end{array}$ \\
\hline Coach & $\begin{array}{l}\text { Wisdom (as a coach can help our next } \\
\text { generation build and re-build from the } \\
\text { scratch) }\end{array}$ \\
\hline Mediator & $\begin{array}{l}\text { Advisor learning and wisdom (facilitate } \\
\text { value co-creation and capability co- } \\
\text { elevation interactions between entities at } \\
\text { multiple scales) }\end{array}$ \\
\hline
\end{tabular}

Children will grow up with their cognitive mediators, and be encouraged by mentors to work on teams to tackle problems with no known solution, and in this way develop a T-shaped skillset and mindset.

Next section briefly explains the proposed framework how these smart machines can interact and boost people's performance.

\section{A Framework of Boosting People's Performance}

Figure 2 demonstrates our proposed framework. In this framework, people interact with CAs through conversations, typing, gesture and multi-model interactions to facilitate value co-creation and capability co-elevation at multiple scales.

Successful interactions enhance people's level of cognition and intelligence that also enhance people's capabilities. Enhanced cognition is considered as the thinking and understanding capabilities generated through the interaction (conversation) between people and CAs. Furthermore, enhanced intelligence is considered as the capabilities of the service system entities namely people and organization augmented with technology as well as capabilities born from interactions among these entities.

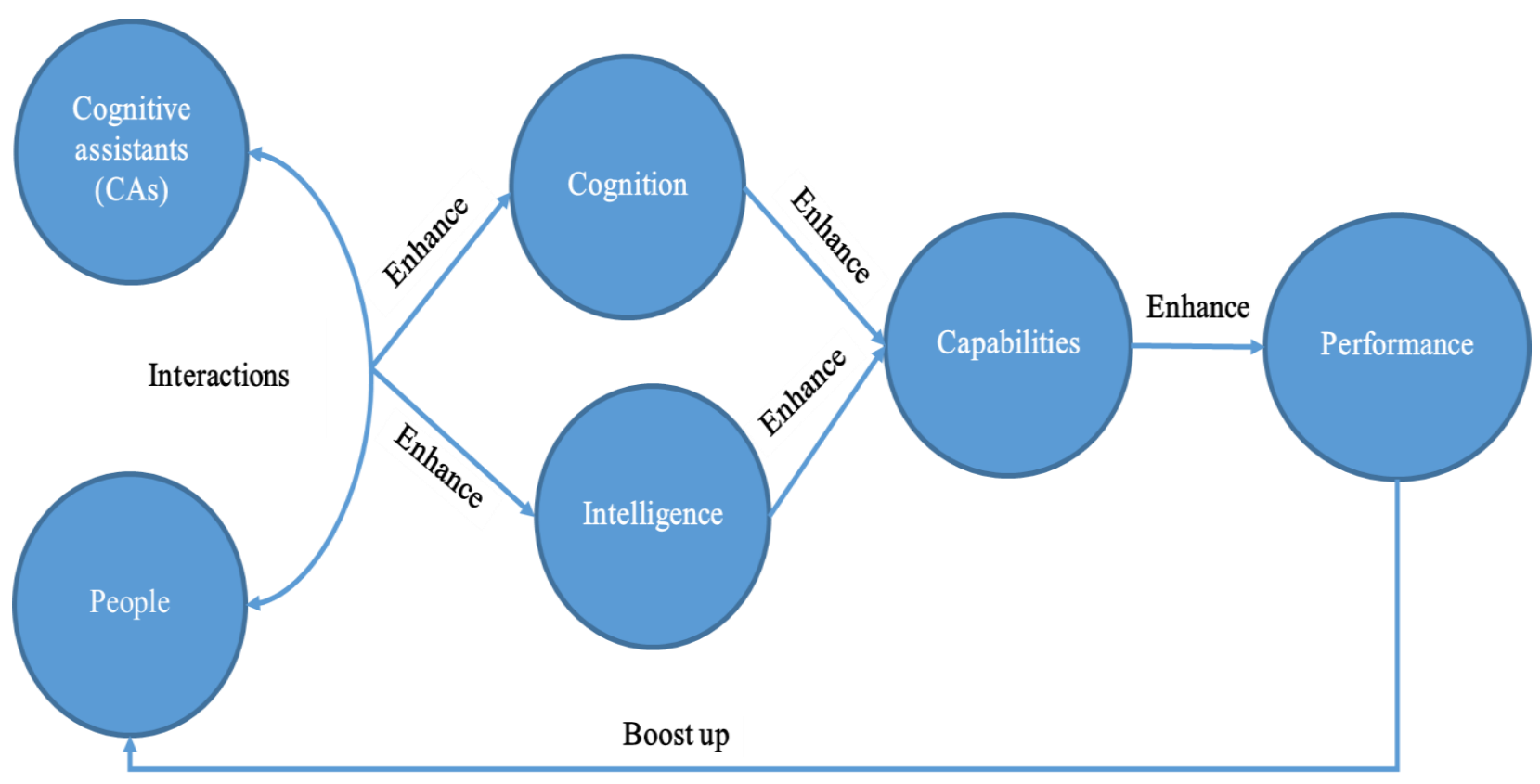

Figure 2. A framework of boosting people's performance

The success of service systems largely depend on the capabilities of the service system entities. The capabilities of the service system impact the ability of entities to compete for collaborators, and succeed coelevating forms of value co-creation. In this regard, enhanced capabilities considered as the ability of people to take smart/wise/right decisions in a complex and conflict situation with leveraging latest information through the interactions with CAs. Finally, successful interactions with CAs also enhance people's 
performance through the enhancement of cognition, intelligence and capabilities.

\section{Research Methodology}

\section{1. Research design}

This is a very new area that requires deep understanding of phenomena from real examples, that's why we used a qualitative case study as a research method [32]. Case studies are well suited to create theoretical constructs, propositions and/or midrange theory [5]. As some scholars view case study research as highly descriptive and stress the social construction of reality [8]. The case study research utilized participant observations and unstructured interviews.

\section{2. Data collection and analysis}

The research was carried out by online interview using open-ended interview protocols. A link of webbased open-ended interview protocol was distributed to the fellows of the First HICSS (Hawaii International Conference on System Sciences) Doctoral Consortium as well as other professional groups during March 2017. A total of 15 responses were received. All the data were analyzed using the techniques of grounded theory [9] that are used in our framework.

We used Corbin and Struss [3] coding method. They identified three types of coding, i.e., open, axial, and selective. Open coding includes an initial pass through the data to come up with candidate concepts for categories. Axial coding includes combining categories into major categories (axial coding). Selective coding is the core category.

The data analysis was done in several steps. First, we converted the online unstructured interview data into a structure form, and then stored them in Excel spreadsheet file. We generated the key concepts for the categories. We then combined the key categories into major categories (broad categories) and generated the core categories after a thorough scanning of the major categories. To ensure anonymity, I1, I2, .................I15 codes were used.

\section{Results of data analysis}

\subsection{Current use of CAs}

The results show that most of respondents use Apple Siri, Google assistant, Facebook messenger as well as Amazon Echo. In addition, one user uses home security device for controlling lights, temperature, air condition and alarming. Most participants responded that they do not prefer to use any CAs because their perception is that they make them lazy.

\subsection{Interactions with CAs}

Most of the participants interact with their CAs by talking with them. One participant also communicates by chatting with her/his CA. One respondent indicated that "I interact by asking questions both for direct information, like about a food recipe or product price. I also ask questions about things I do not know the answer to like how long will it take for sea levels to rise 1 meter" (I7). Another user indicated that "I use Siri periodically to dial my phone numbers as I drive" (I4). Furthermore, another interviewee reported that "the home security is perhaps not really CA, it is more of programming tasks with it and getting data that helps me control things from distance. But I think it is quite close and I could easily see e.g. Siri being connected to it and then making the data more available, more approachable and interactive" (I3).

\subsection{Enhanced cognition}

The results show both negative and positive aspects of using CAs for enhancing users' cognition. In this case, half of the respondents indicated that using CAs enhances their level of cognition. For example, one responded reported that "I think using CAs enhances my cognition. In addition, it helps me to increase my cognition through finding information and providing directions quickly (19). Another user mentioned that "Yes, CAs can enhance my cognition when I am in hurry-talking to Siri can help me" (I8). Furthermore, another user reported that "I think CAs can increase cognition proving information on a specific field" (I3). In contrary, one user indicated that "using CAs not really enhance my cognition. I think there is a problem with CAS detecting words most of the times. So I tend not to use Siri much" (I4).

\subsection{Enhanced intelligence}

The results show mixed opinions from the users. Most of the respondents indicated that using CAs will enhance their level of intelligence. But some of the respondents indicated that using CAs will not enhance their level of intelligence.

In case of enhancing intelligence, one user indicated that "I think using CAs will increase my level of intelligence. I will be able to know something easily" (I5). Another interviewee reported that "Yes, using CAs makes me seem smarter than I am" (I8). Furthermore, 
one user responded that "It does enhance my level of intelligence. It increases my verbal and descriptive level of intelligence" (I11).

On the contrary, some of the users responded that using CAs will not increase their level of intelligence. In this case, one user reported that "using CAs makes me lazier" (I1). Another respondent reported that "using CAs decreases the level of intelligence due to dependency on an external assistant" (I2).

\subsection{Enhanced capabilities}

The result shows that using CAs enhance people's capabilities in terms of getting awareness of options, knowing recognized patterns, criteria to consider in decision making and becoming better at certain routine works. One user reported that "It may help in becoming better at certain (routine) tasks and it can save time for other priorities" (I3). Another interviewee indicated that "using CAs enhances my capabilities. As Siri answers questions and they are always getting more capabilities" (I8). Furthermore, one interviewee reported that "using CAs I can know more options for taking right decision in any complex situation" (I9). Finally, another respondent indicated that "using CAs help me to get awareness about options for right decision" (I15).

\subsection{Enhanced performance}

Most of the respondents indicated that using CAs enhances their performances. For example, one responded reported that "Yes, using CAs enhances my performance. Siri helps me get places faster-so better performance" (I8). Another user indicated that "using CAs helps me to get things done more quickly and efficiently" (I14). In the same way, another respondent described that "I think CAs would improve my performance otherwise I would not use it" (I5). Finally, another user mentioned that "using CAs enhances the quality and efficiency of my performance" (I12).

\subsection{Important issues for success of CAs}

The results of this research show that trust, privacy, accuracy, ease of use and governance are the most important issues for success of current CAs. One user indicated that "trust for sure because I hope that companies are using data to improve CAs but I am not sure for what other purposes companies are using my data (how I use and interact with my CAs). It makes me nervous sometimes that someone records my interaction and then can use it for some unknown purpose that I might not agree with" (I7). Another user indicated that "I would not use Amazon Echo since I do not trust an always-on microphone in me home" (I1). Another interviewee reported that "I think governance. I think that CAs are becoming more and more used by those that are gadget crazed, where as they don't understand the governing entities or policies behind the applications or hardware of CAs. I also think that standards are missing on a lot of new technology" (I4).

\section{Determinants of Measuring Enhanced Performance}

One of the challenges is to how to define and measure the enhanced performance of people as result of their interactions with cognitive assistants. Based on the above founding, we propose a framework for measuring enhanced performance of people through interaction of CAs. Figure 3 depicts the proposed theoretical framework for measuring enhanced performance. In this framework, people interact with their CAs to enhance their level of cognition and intelligence that help them to enhance their capabilities. Finally, the enhanced capabilities of people help them to enhance their performance. Table 4 highlights key constructs to measure enhanced performance using our proposed theoretical framework.

\subsection{People's Interactions with CAs}

According to research, service system entities interact via value propositions [23] [25] [27]. Direct and indirect interactions are the key for co-creation of value. Dialogue is the main mechanism for interactions with several entities (actors) in a service system [1719]. But in case of people and CAs interaction, Lee and Choi [12] describe the human and agent relationship as "give and take" point of view. In addition, Spohrer [21] indicates that CAs are inherently designed to interact with people many times faster than people interact with each other today. Therefore, in this research, we define people - CAs interaction as the conversations between people and CAs to facilitate value co-creation and capability co-elevation at multiple scales.

\subsection{Enhanced cognition}

In general, cognition is the mental action or process of acquiring knowledge and understanding through thought, experience and the senses. We consider enhanced cognition as the thinking and understanding capabilities generated through the interaction (conversation) between people and cognitive assistants. 
We also expect that interactions between people and CAs positively enhance human cognition that also helps to enhance people intelligence. Therefore, we propose that human cognition.

Proposition 4: Enhanced human cognition positively affects the enhancement of people's intelligence.

\section{Proposition 1: People's interactions with CAs}

positively affect the enhancement of

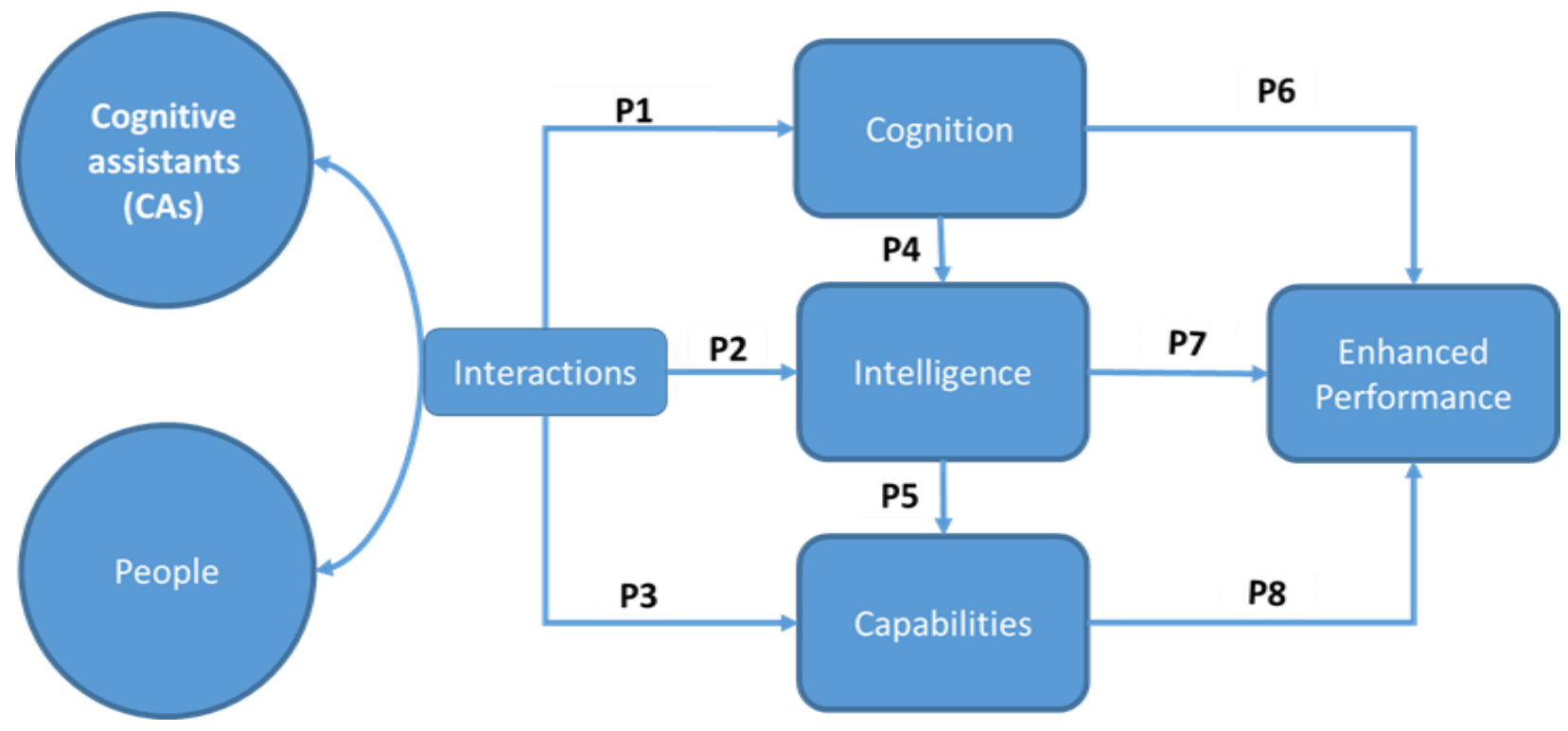

Figure 3. Framework of measuring enhanced performance

Table 4: Highlight of key constructs to measure enhanced performance

\begin{tabular}{|c|c|}
\hline Constructs & Items \\
\hline \multirow{4}{*}{ Interactions with CAs } & I can easily interact with my CAs $(\mathrm{I} 4, \mathrm{I} 7)$ \\
\hline & I can easily talk with my CAs $(\mathrm{I} 4, \mathrm{I} 7)$ \\
\hline & I can easily chat with my CAs $(\mathrm{I} 4, \mathrm{I} 7)$ \\
\hline & I can easily navigate using my CAs $(\mathrm{I} 4, \mathrm{I7})$ \\
\hline \multirow{2}{*}{ Enhanced cognition } & I can easily get information using my CAs (I3, I8, I9) \\
\hline & I can easily get information about people using my CAs $(\mathrm{I} 3, \mathrm{I} 8, \mathrm{I} 9)$ \\
\hline \multirow[b]{2}{*}{ Enhanced intelligence } & I can easily lookup the meaning of words using my CAs $(\mathrm{I} 5, \mathrm{I} 8, \mathrm{I} 11)$ \\
\hline & $\begin{array}{l}\text { I can easily solve numeric calculation problems using my CAs (I5, } \\
\text { I8, I11) }\end{array}$ \\
\hline \multirow{5}{*}{ Enhanced capabilities } & I can easily find new places to eat using my CAs $(I 3, I 8, I 9, I 13, I 15)$ \\
\hline & I can easily dictate memos using my CAs (I3, I8, I9, I13, I15) \\
\hline & $\begin{array}{l}\text { I can easily manage my To Do lists using my CAs (I3, I8, I9, I13, } \\
\text { I15) }\end{array}$ \\
\hline & I can easily find new recipes using my CAs (I3, I8, I9, I13, I15) \\
\hline & I can easily find music to listen to using my CAs (I3, I8, I9, I13, I15) \\
\hline \multirow{5}{*}{ Enhanced performance } & $\begin{array}{l}\text { I can more quickly and efficiently get things done using my CAs (I8, } \\
\text { I12, I14, I15) }\end{array}$ \\
\hline & I can do better quality work using my CAs (I8, I12, I14, I15) \\
\hline & CAs improve my quality of life (I8, I12, I14, I15) \\
\hline & CAs improve my performance on tasks (I8, I12, I14, I15) \\
\hline & CAs improve my productivity $(\mathrm{I} 8, \mathrm{I} 12, \mathrm{I} 14, \mathrm{I} 15)$ \\
\hline
\end{tabular}




\subsection{Enhanced Intelligence}

In general, intelligence is a measure of both learning, the ability to acquire knowledge and skills, and performance, the ability to perform well and survive in complex, and conflict situations [7]. Intelligence is considered as the capabilities of the service system entities namely people and organization augmented with technology as well as capabilities born from interactions among these entities. We expect that the interactions between CAs and people significantly increase the level of people's intelligence. Therefore, we propose:

Proposition 2: People's interactions with CAs positively affect the enhancement of human intelligence. Proposition 5: People's enhanced intelligence positively affects the enhancement of capabilities of people.

\subsection{Enhanced capabilities}

The success of service systems largely depend on the capabilities of the service system entities. The capabilities of the service system impact the ability of entities to compete for collaborators, and succeed coelevating forms of value co-creation [13]. Enhanced capabilities as the ability of people to take smart/wise/right decisions in a complex and conflict situation with leveraging latest information through the interactions with CAs. Therefore, we hypothesize:

Proposition 3: People's interactions with CAs positively affect the enhancement of human capabilities.

\subsection{Enhanced performance}

Enhancing human performance means enhancing human abilities to achieve goals both individually and collectively through the convergence of technologies. Enhancing human performance also hold the promise to make people healthier, wealthier, and wiser as well as to make business more responsive, resilient, and adaptive [22]. In addition, Engelbart [6] asserted that technology could be used to augment human performance to address complex and urgent problems in our society. In the same way, Norman [15] described that technology is an instrument that people has developed to augment their intellect in order to enhance their performance. In this paper, we define enhanced performance as people use CAs to augment interactions and decision making capabilities to solve complex problems. In addition, CAs offer options (appropriate recommendations) associated with a situation that help people to perform well (making wiser decision) to solve problems more efficiently and effectively, overcoming some of the problems of bounded-rationality.

Proposition 6: Enhanced cognition positively moderate the enhanced performance of people.

Proposition 7: Enhanced intelligence positively moderates the enhancement of performance of people Proposition 8: Enhanced capabilities positively moderate the enhancement of people's performance.

\section{Discussion and Future Research Directions}

The main purpose of this research is to understand people's interactions with smart machines, and to develop a framework to boost people's performance through the interaction with cognitive assistants. People's successful interaction with CAs enhances people's cognition, intelligence, and capabilities that help them to boost up their performance in their work. As we know that, collecting data from 15 respondents is a starting point to understand this phenomenon of using cognitive assistants in our personal daily lives.

When these smart machines are growing in our daily lives, we believe that system science, decision science and service science will be greatly benefited from such a research.

This research is not free from limitations. First, we need to further analyze data collected from our pilot study of 15 respondents and further develop theoretical foundations, and framework. And collect data from larger audience to validate the research. Second, in it's current form, we validated our initial draft framework with data from 15 respondents and wit our personal observations. We did not look at the knowledge and skills of these respondents. This was not a controlled study. In the next phase of this research, we hope to address the above items.

\section{Acknowledgement}

This research is supported by the Ministry of Education, Culture, Sports, Science and Technology (Monbukagakusho) scholarship at the Japan Advanced Institute of Science and Technology, Japan.

\section{References}

[1] S. Arbesman, The half-life of facts: why everything we know has an expiration date, Penguin, New York, 2013.

[2] P.B. Baltes and U.M. Staudinger, "Wisdom: a metaheuristic (pragmatic) to orchestrate mind and virtue 
toward excellence, American Psychologist, vol. 55, no. 1, 2000, pp. 122-136.

[3] J. Corbin, and A. Strauss, "Grounded theory research: procedures, cannons and evaluative criteria, Qualitative Sociology, 13(1), 1990, pp. 3-21.

[4] H. Demirkan, C. Bess, J. Spohrer, A. Rayes, D. Allen, and Y. Moghaddam, "Innovation with smart service systems: analytics, big data, cognitive assistance and the internet of everything", Communications of the Association for Information Systems, 37, 2015, pp. 733-752.

[5] K.M. Eisenhardth, and M.E. Graebner, Theory building from cases: opportunities and challenges. Academy of Management Journal, 50, 2007, pp. 25-32.

[6] D.C. Engelbart, "Augmenting human intellect: a conceptual framework", Summary Report, Stanford Research Institute, on Contract AF 49(638)-1024, October 1962, pp. 1134.

[7] H. Gardner, Frames of mind: the theory of multiple intelligence, Basic Books, New York, 1983.

[8] J.H. Gephart, "Qualitative research and the Academy of Management Journal", Academy of Management Journal, 47, 2004, pp. 554-562.

[9] B.G. Glaser, and A.L. Strauss, The discovery of grounded theory: strategies for qualitative research, Aldine De Druyter, New York, 1967.

[10] B.F. Jones, "The burden of knowledge and the 'death of the renaissance man': is innovation getting harder?” NBER Working Paper, no. 11360, May 2005.

[11] J.E. Kelly III and S. Hamm, Smart machines: IBM's Watson and the era of cognitive computing, Columbia University Press, New York, USA, 2013.

[12] S.Y. Lee, and J. Choi, "Enhancing user experience with conversational agent for movie recommendation: effects of self-disclosure and reciprocity", International Journal of Human-Computer Studies, 103(July), 2017, pp. 95-105.

[13] J.G. March, "Exploration and exploitation in organizational learning”, Organization Science, 2(1), 1991, pp. 71-87.

[14] I. Nonaka and H. Takeuchi, "The wise leader", Harvard Business Review, vol. 89, 2011, pp. 58-69.

[15] D.A. Norman, Things that make us smart: defending human attributes in the age of the machine, Basic Books, New York, 1993.

[16] A. Pentland, Honest signals: how to they shape our world, The MIT Press, Massachusetts, 2008.

[17] C.K. Prahalad, and V. Ramaswamy, "Co-creation experiences: the next practice in value creation", Journal of Interactive Marketing, 18(3), 2004, pp. 5-14.

[18] V. Ramaswamy, "Co-creating value through customers' experiences: the Nike case", Strategy \& Leadership, 36(5), 2008, pp. 9-14.

[19] V. Ramaswamy, "Co-creation of value -towards an expanded paradigm of value creation", Marketing Review ST. Gallen, 26(6), 2009, pp. 11-17.

[20] H.A. Simon, Administrative behavior: a study of decision-making processes in administrative organizations, The Free Press, New York, 1997.

[21] J. Spohrer, Innovation for jobs with cognitive assistants: a service science perspective, In Disrupting Unemployment (Ed. Nordfors, Cerf and Senges), Ewing Marion Kauffman Foundation, Missouri, USA, 2016, pp. 157-174.
[22] J.C. Spohrer, and D.C. Engelbart, "Converging technologies for enhancing human performance: science and business perspectives", Annals of the New York Academy of Sciences, 1013(1), 2004, pp. 50-82.

[23] J.C. Spohrer, and P.P. Maglio, Toward a science of service systems, In Handbook of service science, Springer, US, 2010, pp. 157-194).

[24] J. Spohrer, and G. Banavar, "Cognition as a service: an industry perspective", AI Magazine, vol. 36, no. 4, 2015, pp. 71-86.

[25] J. Spohrer, and P.P. Maglio, "Fundamentals of service science", Journal of the Academy of Marketing Science, 26(1), 2008, pp. 18-20.

[26] J. Spohrer, and M.A.K. Siddike, The future of cognitive mediators: tool, assistant, collaborator, coach. Peter Lang Publish (in press), New York, 2017.

[27] J. Spohrer, and P.P. Maglio, Service science: toward a smarter planet, In Service Engineering, ed. Karwowski \& Salvendy, Wiley. New York, NY, 2009.

[28] J. Spohrer, C. Bassano, P. Piciocchi, and M.A.K. Siddike, What makes a system smart? wise? Advances in The Human Side of Service Engineering, 2017, pp. 27-31.

[29] J. Spohrer, A. Giuiusa, H. Demirkan and D. Ing, "Service science: reframing progress with universities", Systems Research and Behavioral Science, vol. 30, no. 5, 2013, pp. 561-569.

[30] J. Spohrer, M.A.K. Siddike, and Y. Kohda, "Rebuilding evolution: a service science perspective", In Proceedings of the 50th Hawaii International Conference on System Sciences, Hilton Waikoloa Village, Hawaii 4-7 January, 2017, pp. 1663-1672.

[31] R. J. Sternberg, Wisdom, intelligence and creativity synthesized, Cambridge University Press, New York, 2003.

[32] R. K. Yin, Case study research: design and methods $\left(5^{\text {th }}\right.$ ed.), Sage, London, 2014. 